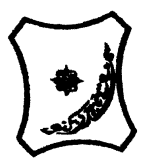

Bayero Journal of Pure and Applied Sciences, 10(1): 570 - 574

ISSN $2006-6996$

\title{
COMPARATIVE STUDY OF PERCENTAGE YEILD AND SOLUBILTY OF CHITOSAN EXTRACTED FROM MALE AND FEMALE SHORT-HORNED GRASSHOPPER
}

\author{
${ }^{1}$ Sani, M. G., ${ }^{1}$ Bashir, U. G. and ${ }^{2}$ Bawa, A. A. \\ ${ }^{1}$ Department of Pure and Industrial Chemistry, Faculty of Science, Bayero University Kano. \\ P.M.B. 4011 Kano State, Nigeria \\ ${ }^{2}$ Kano State College Arts and Science, P.M.B. 3145 Kano State, Nigeria \\ Corresponding Author: bashirgumel69@gmail.com; Tel. 08066606828, 08036350832
}

\begin{abstract}
Chitin was extracted from the exoskeleton of the short-horned grasshopper (Schistocerca gregaria). Chitosan was then obtained by alkaline deacetylation of chitin and physicochemically characterized using Fourier Transformed Infra red Spectroscopy (FTIR) and scanning electron microscope (SEM). Chitin and chitosan were extracted using the conventional Acid-base reaction methods. The FTIR Spectroscopy of the chitosan from male and female short-horned grasshopper revealed that the obtained chitosan is in alpha (a) polymorphic form. This fact was confirmed by the scanning electron microscope (SEM) which shows the morphology of the chitosans from both species of the grasshopper. Solubility test of the extracts have shown that male grasshopper chitosan is much more soluble in $10 \%$ acetic acid than the corresponding female grasshopper chitosan obtained under the same experimental conditions.
\end{abstract}

KEY WORDS: Chitin, chitosan, Deacetylation, solubility and percentage yield.

\section{INTRODUCTION}

Chitin, the source of chitosan is a natural polysaccharide and the second most abundant biopolymer after cellulose. It is a long chain polymer composed of (1-4)-linked 2-acetamido2-deoxy B-D-glucose.

Generally, chitin is found in the exoskeleton of arthropods (crustaceans, insects, mariapods and arachnids) (Murat et al., 2014). The natural biopolymer occur naturally in three polymorphic forms with different orientation of the microfibrils known as alpha (a), beta $(B)$ and gamma $(\gamma)$ chitin.

Chitin and chitosan have excellent properties, such as biodegradability, biocompatibility, non toxicity and absorption (Judson, 2013). Chitin and chitosan are now attracting great interest because of their wide range of potential applications in the field of Biotechnology, medicine and pharmacology, agriculture, textiles, cosmetics and waste water treatments (Narguess et al., 2004). Recently, chitin and its derivatives have found wider application as polyelectrolyte properties, gel forming ability, high adsorption capacity, Healthy weight loss fills and wound healing applications, matrix removal and for immobilization of biomolecules, support of biosensors, heavy metals removal and removal of radioactive waste. This has gained the attention of many researchers and made them search for more new chitin source. Crab, shrimp and cray fish has been prepared for commercial production of chitin, but new sources like insects that includes grasshopper, crickets, locusts, katydids and their related species are a great area of research.

Grasshoppers are widely spread throughout the World, approximately 20000 described orthopteran species have been acknowledged Worldwide up to now, but these organisms have been highly ignored with regards to chitin structure (Murat et al., 2015).

The aim of this study is to isolate chitin from local insects sources in Nigeria (short-horned grasshopper). The chitin obtained was deaceylated to the more useful chitosan and the physico-chemical properties of chitosan was determined using FTIR analysis and scanning electron microscope (SEM).

\section{MATERIALS AND METHODS}

The insect species used for extraction of chitin and chitosan is male and female short-horned grasshopper (Schistocerca gregaria) was analytically grade.

Sodium hydroxide, Hydrochloric acid and Acetic acid were used. 
Sample Preparation.

The insects were sorted into male and female species and their exoskeleton was prepared for the study by first removing the internal organs and scraping any loosed tissue. The materials was then washed, dried and crushed into powder using mortar and pistol.
Isolation of Chitin and synthesis of Chitosan The exoskeleton does not exist as stand alone biopolymer, but rather in a conglomeration with other biomaterials, mainly proteins, lipids and inorganic salts. The extraction processes is illustrated in the reaction scheme 1 :
Exoskeleton of short-horned grasshopper $+5 \% \mathrm{HCl}$
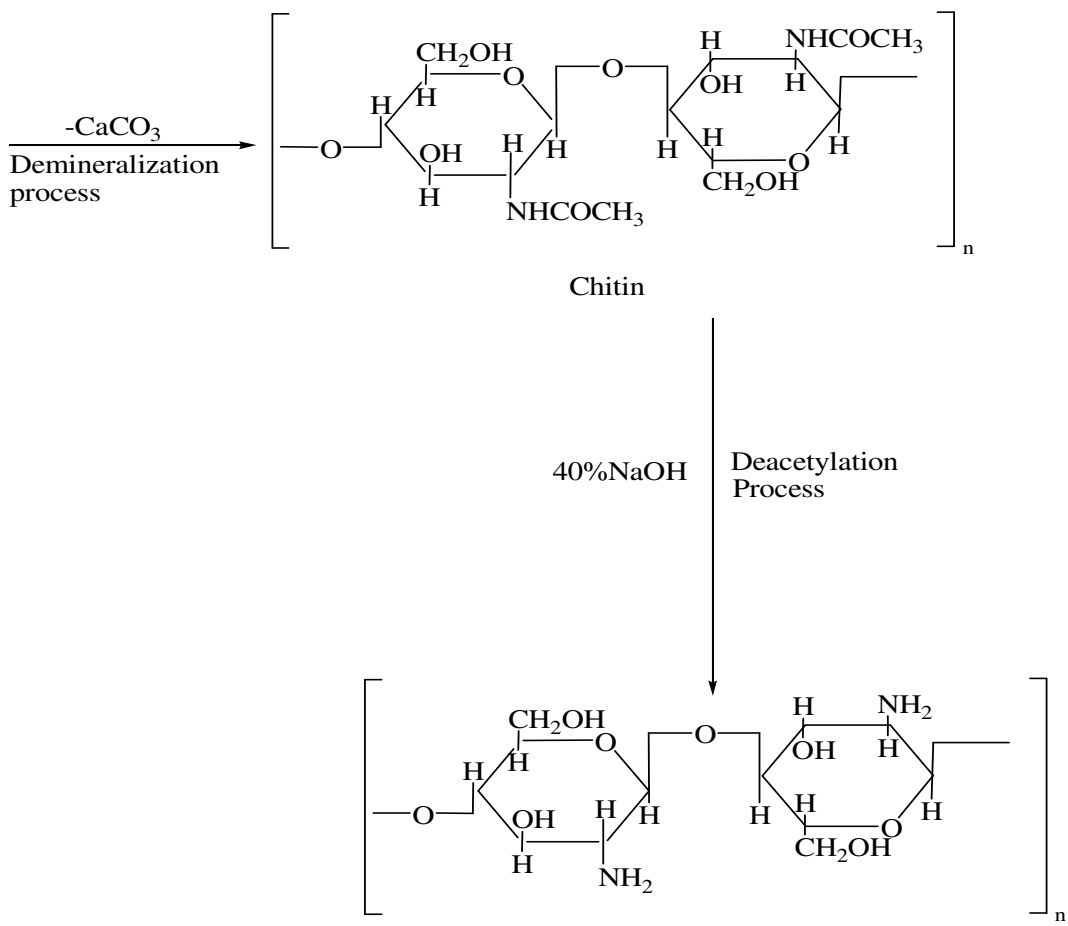

Chitosan

Scheme 1: Reaction scheme for the extraction of chitosan from male and female short horned grasshopper

In the process, treatment of the exoskeleton with $5 \% \mathrm{HCl}$ dissolves the calcium salts and lipids and other minor inorganic constituents to yield chitin. Treating the resultant chitin with $40 \% \mathrm{NaOH}$ solution at $120^{\circ} \mathrm{C}$ for 2 hours with stirring for homogeneity, yield the desired chitosan (Narguess et al., 2015).

During the alkaline treatment, a concomitant hydrolysis of the acetamido groups of chitin takes place, this results in the formation of Chitosan. The resultant chitosan was washed thoroughly with distilled water and oven dried at $50{ }^{\circ} \mathrm{C}$ for 24 hours (Rawda and Hadeer, 2015).

The extracts was purified by dissolving in $2 \%$ acetic acid and re precipitated out using 20\% $\mathrm{NaOH}$ solution. The pure chitosan was then washed to neutrality and oven dried for 24 hours and then grounded into powder with an electrical blender.

\section{Characterization}

The chitosan extracted from the alkaline deacetylation of the chitin obtained from both sexes of the short-horned grasshopper was characterized using the following processes:

\section{FTIR Spectroscopy}

Chitosan powder from both male and female grasshopper were prepared for FTIR analysis, using FTIR Spectrocopy over the range of 400$4000 \mathrm{~cm}^{-1}$ spectral region.

The absorbance of the spectral peak observed was compared with the absorbance of the reference peak to determine the degree of deacetylation of the sample ( Abdulwadud et al., 2013).

Scanning electron microscope (SEM);

Scanning electron microscope was used to examine the surface morphology of the chitosan powder for both male and female species.

\section{Degree of deacetylation}

The degree of deacetylation (DD) of chitosan obtained from male and female short-horned grasshopper was determined by comparing the absorbance of the Amide peak to that of the Hydroxyl group. This was used to calculate the degree of deacetylation of the samples using equation (1) 
$\mathrm{DD}=100-\left[\mathrm{A}_{1659} / \mathrm{A}_{3428} \times \frac{100}{1.33}\right.$

Where A1659 and A3428, are the absolute heights of the absorption bands of Amide and Hydroxyl groups respectively and 1.33 is a constant (Marianna and Svetlana, 2015).

\section{Solubility of Chitosan}

The chitosan samples from both male and female short horned grasshopper were tested for solubility in aqueous solution of acetic acid. $1 \mathrm{~g}$ of chitosan powder from male and female grasshopper was weighted and labeled as CHTM and CHTF for male and female chitosan in a centrifuge tubes (of known weight) respectively and dissolved with $10 \mathrm{ml}$ of $10 \%$ acetic acid solution for 30minutes using an incubator shaker at $240 \mathrm{rpm}$ and $25^{\circ} \mathrm{C}$. The solution in was then immersed in boiling water bath for 10minutes, cooled to room temperature and then centrifuged at $10,000 \mathrm{rmp}$ for 10 minutes. The supernatants was decanted and the undissolved particles washed in distilled water and then centrifuged against at 10,000rmp and the supernatant was removed and the undissolved particles were dried at $60^{\circ} \mathrm{C}$ for 24hrs: finally, weight and the percentage solubility was calculated as:

$$
\text { Percentage solubility }=\frac{\text { Initial weight of tube }+ \text { chitosan }- \text { final weight tube }+ \text { chitosan }}{\text { Initial weight of tube }+ \text { chitosan }- \text { Initial weight of tube }}
$$

RESULTS AND DISCUSSION

Chitosan Yield:

The yielding proportion of Chitosan obtained from the male and female grasshopper at various stages of extraction are depicted in Table 1.

Table 1: Experimental yield of each stage for the extraction of Chitosan from male and female short-horned grasshopper

\begin{tabular}{lcc}
\hline Category & Male & Female \\
\hline Exoskeleton $(\mathrm{g})$ & 50 & 50 \\
Stage 1: \% weight of the sample after demineralization & $40 \mathrm{~g}(80 \%)$ & $34.23 \mathrm{~g}(68.46 \%)$ \\
Stage 2: \% weight of the product remaining after & $18.2 \mathrm{~g}(45.5 \%)$ & $9.22 \mathrm{~g}(23.64 \%)$ \\
deacetylation & & \\
$\%$ weight of the final product (Chitosan) & $36.4 \%$ & $18.44 \%$ \\
\hline
\end{tabular}

From table 1, it was established that the extraction processes was initiated by demineralization process, where $(20$ and $31.54 \%$ ) of the samples were removed as minerals (comprising $\mathrm{CaCO}_{3}$ and other minor inorganic salts). While the second stage which is deacetylation process removes the acetyl groups by the action of strong alkaline solution of Sodium hydroxide., at a very high temperature, yielding $45 \%$ and $23.64 \%$ of chitin to chitosan and 36.4 and $18,44 \%$ of the exoskeleton to chitosandry weight was recovered as chitosan from male and female FOURIER TRANSFORMED INFRARED SPECTROSCOPY (FTIR)

The FTIR spectrum of the male and female short-horned grasshopper (CHTM \& CHTF). Displayed the following absorption bands:

These absorption bands were observed in the spectrum of the male grasshopper Chitosan (CHTM) and the female grasshopper Chitosan (CHTF) and they are the major characteristics of Chitosan as shown in Figure 1 and Table 2 respectively, (Rawdar and Hadeer,2015). grasshopper respectively (Murat etal,2015).

Table 2: absorption bands and Chemical groups in male and female short-horned grasshopper Chitosan (CHTM and CHTF).

\begin{tabular}{lllllll}
\hline Functional group & $\mathrm{OH}$ & $\mathrm{NH}$ & $\mathrm{C}=\mathrm{O}$ & $\mathrm{NH}_{2}$ & $\mathrm{CH}_{2}$ & $\mathrm{CH}$ \\
\hline Absorption band of CHTM $\left(\mathrm{cm}^{-1}\right)$ & 3217 & 3257 & 1659 & 1547 & 2918,2873 & 1424,1380 \\
Absorption band of CHTF $\left(\mathrm{cm}^{-1}\right)$ & 3428 & 3264 & 1659 & 1547 & 2918,2873 & 1424,1380 \\
\hline
\end{tabular}




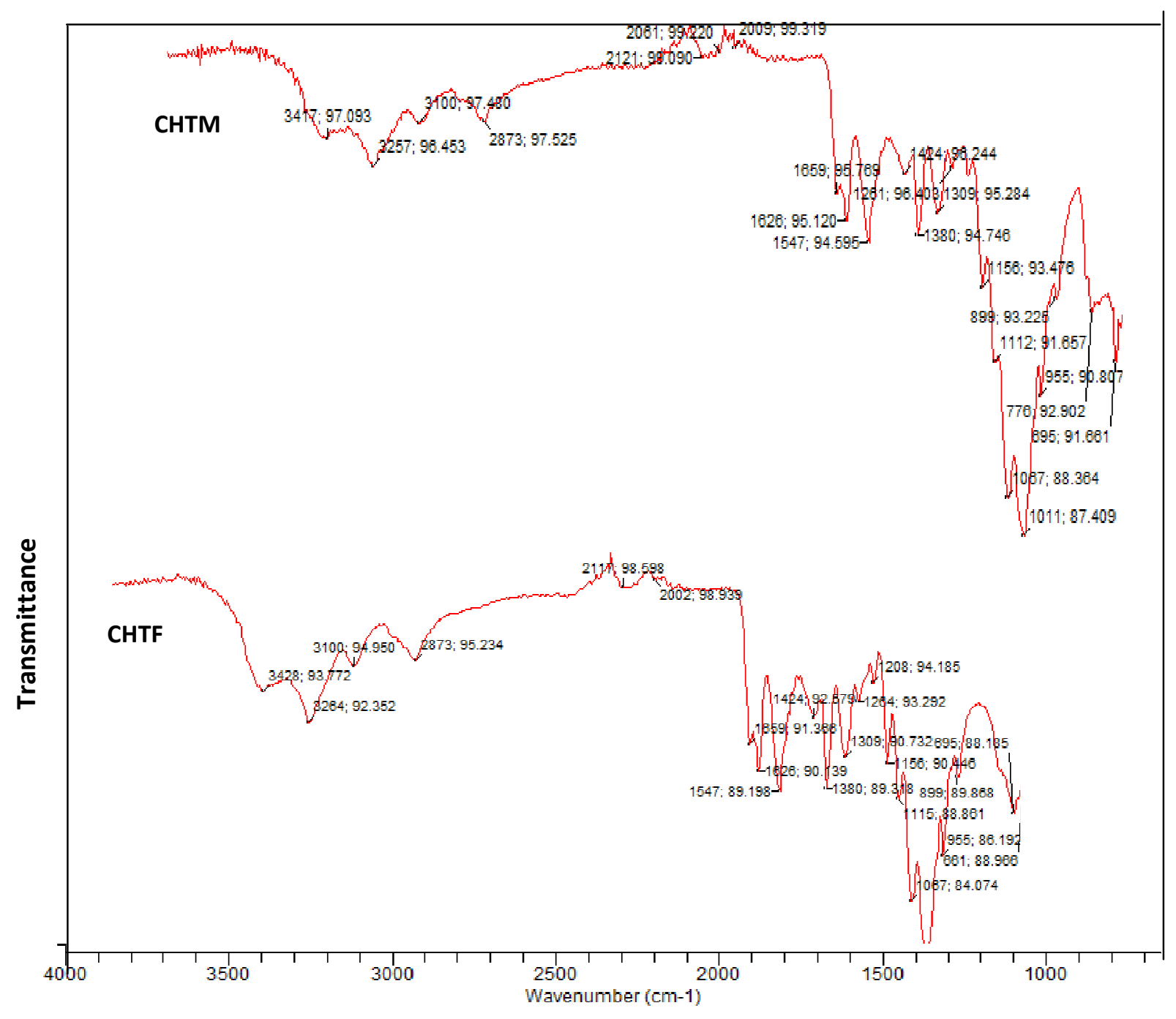

\section{Scanning Electron Microscope (SEM)}

For better understanding of the surface morphology of the chitosan obtained from male and female short-horned grasshopper, the samples were examined using scanning electron microscope. The results shows that male chitosan was found to be porous as shown in figure 2. The female chitosan was expected to have the same surface structure but differ, despite that the same chemical procedure used during the extraction. This could probably result from the high protein content of the female specie which maintain the exoskeleton non-porous (Murat et al.,2015)
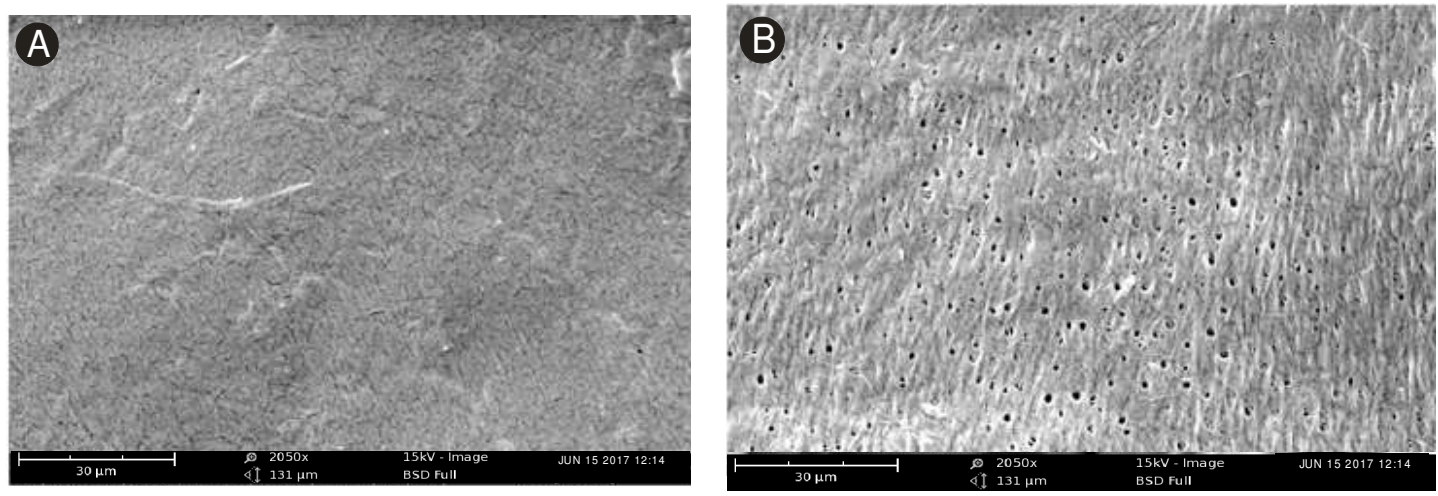


\section{Special Conference Edition November, 2017}

Degree of deacetylation:

The degree of deacetylation of the resultant chitosan, obtained from male and female grasshopper have shown that male specie had the highest yield and hence have high degree of deacetylation of $98.63 \%$. While the female grasshopper has $89.43 \%$ (Murat et al., 2015). It was established that the degree of deacetylation (DD) of the chitosan extracted from male and female grasshopper are comparable (98-89\%) and are higher than that of commercial chitosan from Shrimp, prawn, or crab ( Narguess, 2015).

Solubility

Table 3: Experimental analysis of the percentage solubility test of Chitosan from male and female short-horned grasshopper

\begin{tabular}{lccccc}
\multicolumn{1}{c}{ Sample } & CHTM & & \multicolumn{2}{c}{ CHTF } \\
& $(\mathbf{g})$ & & (g) & $\%$ \\
\hline Chitosan powder & 1.00 & & - & 1.00 & - \\
\% solubility & 0.752 & & 75.2 & 0.463 & 46.3 \\
\hline
\end{tabular}

From table 3 , it was established that male chitosan is more soluble in dilute acetic acid with about $75 \%$ solubility, while female chitosan have $46.34 \%$. The result is in conformity with Murat et al.(2015). However, it was postulated that solubility below $87.0 \%$ is considered as low solubility. Thus, lower solubility could be due to incomplete removal of the acetyl group. Since the chemical basis of the method was based on the reaction with amino group, the presence of protein contaminants remaining in the sample during analysis could interfered with the results.

\section{CONCLUSION}

The study examined the difference between the male and female grasshopper chitosan extracted under the same experimental

\section{REFERENCESS:}

Abdulwadud A., Muhammed T. I., Surajuddeen A., Abubakar J. M., Alero O. A (2013).

Extraction and characterization of chitin and chitosan from Mussel shell. Civil and Environmental research 3(2). www.iiste.org

Judson H.W., Shy. L, (2013); Synthesis and characterization of Chitosan from Shrimps shells. BSc. Engineering (Hons.) Material and Manufacturing Engineering, Faculty of Engineering and Science, University Tunku-Abdul Rahman.

Kaewboonruage, S., Phatrabuddha N., Sawangwong, P. and Pitaksanurat S. (2016). Comparative studies on the extraction of Chitin- Chitosan from Golden Apple snail shells at the Control field. IOSR Journal of polymer and Textile Engineering (IOSR-JPTE), 3 (1):34-41.

Marianna L. and Svetlana C., (2005). Preparation of Chitosan Powder and conditions, in relation to percentage yield and solubility in acetic acid solution. The results revealed that male grasshopper had the greatest yield of chitosan $(46.4 \%)$, while the female had only $18.44 \%$. It was established that the degree of deacetylation (DD) of the chitosan extracted from male and female grasshopper are comparable (98-89\%).Solubility test revealed that male grasshopper Chitosan had the highest percentage solubility of $75.2 \%$ than the female grasshopper chitosan with 46.3\%. Eventhough the male spacies have $75.2 \%$, yet the chitosan consist of protein that should be deprotienated, hence deportinization process should be carried out before deacetylation if high solubility is to be achieved.

Investigation of its properties. Proc. Estonian Acad. Sci. Chem, 55(2):78-84.

Murat K., Evaldas L. Radvile N., Idris S., Gulsin A., Abas M., Talat B., Esran C. and Betal B, (2015). Differenciations of Chituin Content and Surface Morphologies of Chitins Extracted from male and female grasshopper species. PLOS

ONE/DOI:10.1371/Journal.pone.011553 1

Narguess H. M., Emtithal A., Taher S., Gamal R. S. and Ahmed H. M. E. (2015). Isolation and Characterization of Chitosan from different local Insects in Egypt. International Journal of Biological Macromolecules. (82):871-877.

Rawda M. B. and Hadeer I. M., (2015). Chitin extraction, composition of different six Insects species and their comparable characteristics with that of the Shrimp. Journal of American Science. 11(6):127134. 